\title{
Une galerie consacrée à l'art carcéral Daniel Lamoureux*
}

\section{IL ÉTAIT UNE FOIS...}

L'aventure commence à l'hiver 1980. Elle s'étendra sur huit années, soit jusqu'à l'automne 1988, essentiellement au Québec. Elle impliquera des dizaines de bénévoles, des peintres, des sculpteurs, des comédiens, des musiciens, des auteurs, des poètes, la plupart étant ou ayant été détenus. À leur art on attribuera dès lors le qualificatif 'carcéral' : ce printemps-là le concept d'art carcéral naît au Québec. Il ouvrira une fenêtre sur la prison, une vue du dedans qui surprendra des milliers de Québécoises et de Québécois. Car il s'agit bien, on l'aura deviné, d'un projet autant social qu'artistique.

C'était le 11 février 1980, à 20 heures, rue Isabella, chez Andrée Lachapelle. Il y avait là outre l'hôtesse : Marie Boissonnault, Ginette Potvin, Michel Brière, Raymond Goyer, Daniel Lamoureux et Alexandre Zelkine. Ce soir-là fut conçu le MoUvement POUR LA DIFFUSION DES ARTS CARCÉRAUX DU QuÉBEC (MDACQ), qui naquit officiellement le 2 mai suivant et fut enregistré à Québec le 9 juin 1980. Sa mission, tant sociale que culturelle, s'articulait sur trois plans :

- contribuer à stimuler la créativité artistique chez les détenu(e)s et les ex-détenu(e)s ;

- contribuer à la diffusion d'œuvres littéraires, musicales, picturales, photographiques ou audiovisuelles traitant de la prison, des prisonniers, ou de matières connexes; et

- contribuer à la diffusion de productions littéraires ou artistiques de détenus(e)s ou d'ex-détenu(e)s.

Sur les neuf années suivantes ce mouvement allait :

- réaliser dans les principales villes du Québec plusieurs centaines d'événements qui se déclinent en termes d'expositions (solos ou collectives) de sculptures, de peintures, d'artisanat ou de photographies, de spectacles de musique, de chansons, de théâtre, de poésie, de lancements de livres, de conférences, de présentation de vidéos ou de diaporamas, de coordination de plusieurs éditions de la Semaine du prisonnier et l'organisation d'un bon nombre de leurs activités respectives ; 
- se doter d'une bannière exceptionnelle en la Galerie Maximum, modeste local situé au 123 ouest, avenue du Mont-Royal, à Montréal, qui deviendra au fil des ans le lieu de rendez-vous des groupes et individus intéressés par l'art carcéral ;

- mettre sur pied le groupe Évasion, constitué principalement de Sylvie Painchaud et de Réjean Bébé Ferland, qui allaient ponctuer la tournée de nombreuses villes du Québec de leurs prestations énergiques et touchantes;

- participer à de nombreuses conférences de presse, émissions de radio ou de télévision, reportages et articles parus dans des journaux ou des revues; et

- encourager de manière tangible la production d'œuvres d'art chez les artistes suivants, notamment via leur exposition publique : Yvon Auclair, Rolland Berthiaume, Bill Bickerkike, Marcel Blanchette, Jean-Mario Cédilotte, Louis-Philippe Chamberland, Normand Champagne, Alain Champoux, André Côté, Florent Cousineau, Marcel Crevier, François De Lucy, Abdellatif Derkaoui, Louyse Doucet, Pierre-A. Dupuis, Martine Fourcand, Chin Kon Fong, Jean-Louis Lapierre, Jean Le Breton, Guy Lépine, Donald Milliard, William Papier, PDG (Pedneault, Desautels, Gaudreau), Miguel Angel Polanco, Lise Rose, Yvon Roy, Michel Soly, Marcel Soucy, Yves Thrace, Jacques Tourigny, René Tremblay, Maxime Uomobono, Armand Vaillancourt, Mario Viboux et Chu Hong Wai.

\section{LES ANNÉES 1980}

Ces événements remarquables s'inscrivent dans le cadre d'une décennie d'effervescence à la fois culturelle et politique, qui vit s'épanouir un Québec ouvert sur le monde et fort de ses racines identitaires, fier de sa jeunesse et confiant en son devenir économique. Les causes du sabordage de la GALERIE Maximum en 1988 sont bien sûr liées aux conséquences du krach d'octobre 1987 et à l'austérité qui s'ensuivit au Canada, mais surtout au sentiment de lassitude des piliers du MDACQ, qui entreprirent dès lors des carrières personnalisées. 


\section{UNE GALERIE VIRTUELLE}

Quelque trente ans plus tard, une poignée des pionnières et pionniers de l'édition initiale de la GALERIE MAXIMUM relancent bénévolement le projet, un projet de galerie virtuelle cette fois, sans limite de langue, d'espace, de main-d'œuvre. Dans cette perspective un site web a été créé durant l'été 2015 (www.galeriemaximum.ca). D'ici peu des photographies d'oeuvres d'art carcéral y seront affichées peu importe leur provenance. Les communications reçues de la part de visiteurs via ce site seront retransmises intégralement à l'artiste concerné, par courriel ou par courrier. Aucune commission ne sera prélevée par la GALERIE MAXIMUM s'il y a vente, aucun coût d'affichage ne sera facturé aux artistes, aucun frais ne leur sera imposé pour photographier leurs œuvres. Trois conditions réglementeront les participations artistiques :

- les exposantes ou exposants sont détenu(e)s ou ex-détenu(e)s

- les œuvres affichées, ou leurs titres, ne contreviennent pas, dans leur nature ou leur caractère, aux lois canadiennes

- les auteurs doivent préalablement signer une autorisation d'affichage de leurs œuvres sur le site de la GALERIE MAXIMUM conformément à la lettre et à l'esprit de la loi sur les droits d'auteur, accepter qu'un professionnel en prenne des photographies, et dégager la GALERIE MАхIмUм de toute responsabilité à l'égard de l'usage que pourraient éventuellement en faire des internautes mal intentionnés.

Il va de soi que l'actualisation de ce projet reste conditionnelle à son financement, si minime soit-il, par un bailleur de fonds gouvernemental ou privé.

\section{NOTES}

* Dans ce document, le masculin désigne tant les femmes que les hommes et, le cas échéant, n'est utilisé que pour alléger le texte.

\section{NOTE BIOGRAPHIQUE}

Daniel Lamoureux naît dans les Laurentides en 1945. En 1970, une arrestation interrompt ses études universitaires et on le condamne à 20 ans. 
En 1980, peu après une libération conditionnelle, il reprend ses études et fonde le Mouvement pour la diffusion des arts carcéraux du Québec, dont sera issue depuis 1983 la Galerie Maximum. Parallèlement, il publie Y a rien là ! et sa traduction No Big Deal, une pièce de théâtre créée en 1977 par des détenus du pénitencier Archambault, un établissement à sécurité maximale où il séjourna durant six ans. Il entreprend dès lors une carrière de 30 ans en gestion d'organismes communautaires oeuvrant dans les domaines des arts, des jeunes sans-abri et surtout de la représentation politique. Dans le cadre de cette carrière il résidera à Montréal, Yellowknife et Iqaluit. De retour dans sa région natale aux côtés de ses garçons âgés (début 2015) de 9 et 10 ans, il relance une Galerie Maximum à la fois virtuelle et internationale, ce qui est l'objet de l'article publié dans le présent numéro. On peut joindre dès maintenant la Galerie Maximum en s'adressant à Daniel Lamoureux par courriel (galeriemaximum@hotmail.ca) ou par la poste à l'adresse suivante:

Galerie Maximum

Casier postal 49

Saint-Sauveur, Québec

J0R 1R0

Canada 


\section{PHOTOS PERTINENTES}

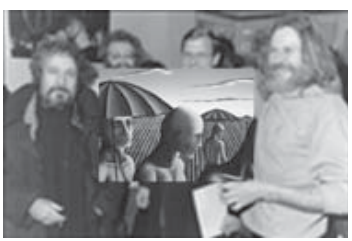

Vittorio Fiorucci, designer graphique, Pierre-Paul Geoffroy, directeur de la GALERIE Maximum, André Gourd, Québecor,

Armand Vaillancourt, sculpteur, au vernissage du 31 janvier 1984

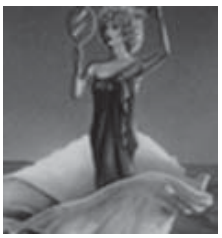

Yvon Roy, février 1986 : Aubain téléphonique

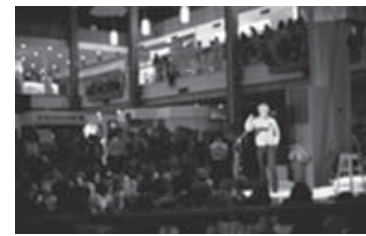

Yvon Deschamps, spectacle collectif On s'en sort, Fort Chambly, le 14 avril 1986 4 juin 1982, Place Laurier, Québec

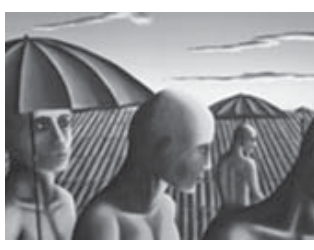

Marcel Blanchette, 1985 : Les parapluies acides
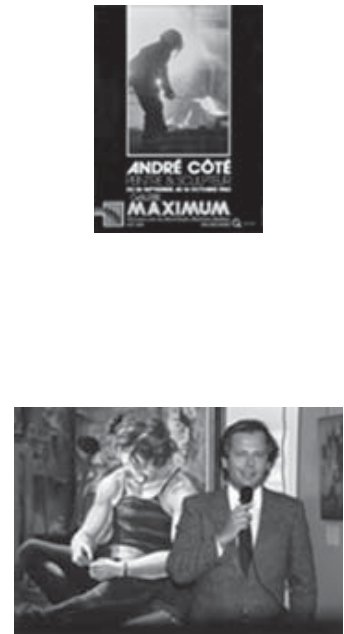

Le ministre Gérard Latulippe,

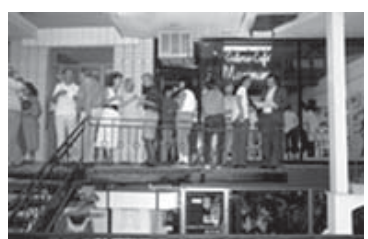

GaLerie MaXimum, 1987 : le ministre Gérald Godin, le poète Michel Bujold, Daniel Lamoureux, directeur général

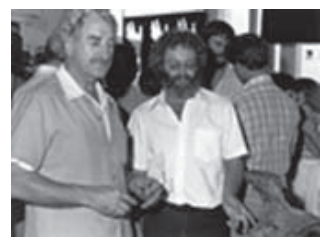

Michel Chartrand et Pierre-Paul Geoffroy, le 31 janvier 1984

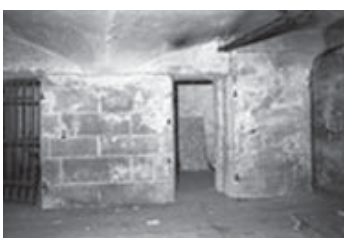

Première prison à Québec 1812-1867 


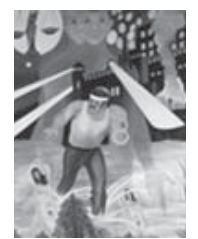

Louis-Philippe Chamberland, novembre 1985 : Évasion

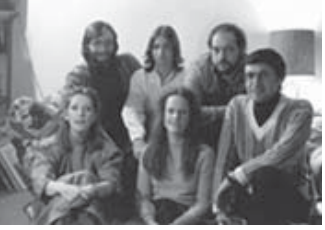

11 février 1980 : les

fondatrices et fondateurs du MDACQ et de la GALERIE

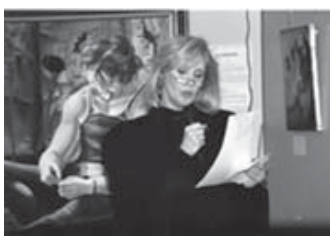

6 septembre 1983 :

la présidente Andrée Lachapelle inaugure la GALERIE MAXIMUM

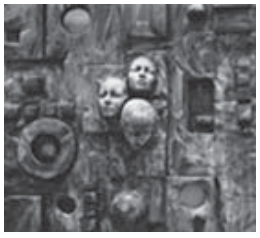

Rolland Berthiaume, avril 1982

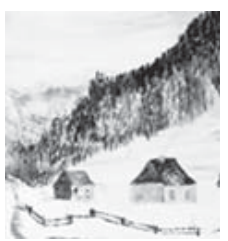

Florent Cousineau, décembre 1983 :

Solitude

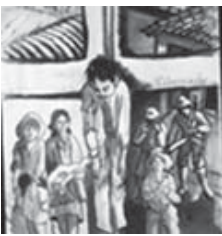

Miguel Angel Polanco, mars 1986 :

Vendredi saint salvadorien

MaXimum 